\title{
Models of Codend Size Selection
}

\author{
R. J. Fryer \\ SOAEFD Marine Laboratory, P. O. Box 101, Victoria Road \\ Aberdeen, AB11 9DB, Scotland, United Kingdom \\ and \\ J. G. Shepherd ${ }^{1}$ \\ MAFF Fisheries Laboratory, Pakefield Road, Lowestoft \\ Suffolk, NR33 OHT, England, United Kingdom
}

\begin{abstract}
Different models relating codend selectivity to net variables such as mesh size, extension length and codend diameter often fit experimental data almost equally well. The choice of an appropriate model must then be determined by other considerations. This is particularly important when, for management reasons, it is necessary to extrapolate outside the range of the experimental data. Two families of codend selectivity models were considered. One family was developed using empirical fits to data. The other was developed using arguments about the physical and biological mechanisms which underlie selectivity; this family provides plausible prior selectivity models which might be useful for extrapolation. The fit to experimental data and the consequences of extrapolating each model was investigated. Although an 'empirical' model often provided the best fit, this sometimes gave unrealistic predictions outside the range of experimental data and such a model should not be used for extrapolation. When a plausible prior model gives the best fit to the data, its use is generally recommended. Otherwise, procedures are suggested for balancing the conceptual advantages of a plausible prior model with the empirical evidence for an alternative model.
\end{abstract}

Key words: Fishing gear, gear slectivity, statistical models.

\section{Introduction}

Recent investigations of codend selection have stressed the importance of using appropriate statistical methods in fitting models to data (Fryer, 1991; Millar, 1992, 1993, 1994; Millar and Walsh, 1992) and of considering variables other than mesh size, such as codend diameter and the length of the extension piece (Reeves et al., 1992; Galbraith et al., 1994). However, relatively little attention has been given to the most appropriate functional form to be fitted. A logistic curve is now commonly used to relate retention probability to length (Pope et al., 1975), although recently, asymmetric (Millar, MS 1991) and nonparametric (Millar, 1993) selectivity curves have also been used. Reeves et al. (1992) showed that the parameters of the logistic curve can depend on variables such as mesh size and codend diameter, and that these relationships can be expressed in several ways. These various alternatives may fit the data almost equally well and a choice between them may have to be guided by other consideration; for example, see the discussion by Reeves et al. (1992) on the choice between their equations (5) and (7).
The differences between alternative models may only become apparent when they are extrapolated beyond the range of the data fitted. Formally, of course, such extrapolation is undesirable. Regrettably, however, it is often unavoidable in practice; for example, when considering an increase in mesh size beyond that used in selectivity trials to date. In such circumstances, the known dangers of extrapolation may be reduced by using only models that are 'robust' to extrapolation; that is, models that give 'sensible' predictions, consistent with the available understanding of the underlying physical and biological mechanisms of codend selection. Indeed, models robust to extrapolation may be preferred to other candidate models, even when their fit to experimental data is marginally worse, if the purpose is prediction outside the range of the data. A well known situation in which this occurs is the fitting of polynomial regression models; such models are often useful for describing trends in data, but should rarely be used for extrapolation. The selection of appropriate families of models rarely receives the attention it deserves; Gilchrist (1984) gives a useful discussion of the issues involved.

\footnotetext{
${ }_{1}^{1}$ Present address: University of Southampton, Shackleton Building, Highfield, Southampton, SO17 1BJ.
} 
This paper considers two families of codend selection models, which we loosely call 'empirical' and 'structural'. The empirical models have been used with experimental data to show the effects of mesh size, codend diameter and extension length on selectivity (Reeves et al., 1992; Galbraith et al., 1994). The 'structural' models are developed here using arguments about the underlying physical and biological mechanisms of codend selection and are more directly based on the traditional selectivity concepts of selection factors and ranges. These models are plausible and internally consistent $a$ priori and therefore should provide reasonable, feasible predictions. We first describe the empirical and structural models, and investigate the fit of each model to four selectivity data sets. We then consider the interpretation and application of each model, with particular emphasis on extrapolation. Finally, we discuss the management implications of model selection.

\section{Empirical Models}

Reeves et al. (1992) and Galbraith et al. (1994) describe selectivity trials for haddock, whiting and cod using:

- two seine nets and a single boat trawl with, approximately, mesh sizes of $80-100 \mathrm{~mm}$, extension lengths of $0-13 \mathrm{~m}$ and codend diameters of 2-4 m,

- a pair trawl with mesh sizes of 80-110 mm, codend diameters of 2-4 $\mathrm{m}$, and a constant extension length of about $11 \mathrm{~m}$. the form:

Using these data, they investigated models of

$$
\begin{array}{r}
\text { Model E1 } \log _{e}\left(\frac{p}{1-p}\right)= \\
\alpha_{1}+\alpha_{2} m+\alpha_{3} e+\alpha_{4} \mathrm{c}+ \\
\left(\alpha_{5}+\alpha_{6} m+\alpha_{7} e+\alpha_{8} c\right) l
\end{array}
$$

where $\mathrm{p}, \mathrm{I}, \mathrm{m}, \mathrm{e}, \mathrm{c}$ are retention probability, fish length, mesh size, extension length and codend diameter respectively and $\alpha_{1}, \alpha_{2}, \ldots \alpha_{8}$ are unknown parameters that are species and net dependent. Further, they found two simplifications of this model:

$$
\begin{aligned}
& \text { Model E2 } \log _{e}\left(\frac{p}{1-p}\right)=\alpha_{1}+\alpha_{2} m+\alpha_{3} e+\alpha_{4} c+\alpha_{5} \text { I } \\
& \text { Model E3 } \log _{e}\left(\frac{p}{1-p}\right)=\alpha_{1}+\alpha_{3} e+\alpha_{4} c+\left(\alpha_{5}+\alpha_{6} m\right) \text { I }
\end{aligned}
$$

which both adequately described the selectivity data for all species/net combinations over the range of experimental mesh sizes, extension lengths and codend diameters. Full details are given in the references above.

For comparative purposes, we also consider here a very simple model in which selectivity does not depend on any explanatory variables (other than length): namely,

$$
\text { Model E0 } \log _{e}\left(\frac{p}{1-p}\right)=\alpha_{1}+\alpha_{5}
$$

\section{Structural Models}

The traditional parameterization of a logistic curve for a particular mesh size $m$ (and any other relevant variables) has been in terms of the $50 \%$ retention length $I_{50}$ and the selection range $S R=I_{75}$ $-I_{25}$. It has also been traditional to express the $50 \%$ retention length in scaled form, as the selection factor $S F=I_{50} / \mathrm{m}$. It has not been so common to express the selection range in the equivalent scaled form, as the range factor $R F=S R / m$, but we shall find this useful also. Such non-dimensional scaling is a common procedure in physics and engineering and (although sometimes merely a matter of convenience) often reflects fundamental properties of the system under discussion. This leads to the powerful technique confusingly known as dimensional analysis, much used in fluid dynamics (see, for example, Fender, 1957).

The problem of mesh selection is essentially geometrical. Selectivity can be thought of as the passage of similarly shaped objects of various sizes (fish) through square or diamond shaped (but somewhat deformable) holes. Given a particular net and size composition of fish, what would happen if the size of mesh and the sizes of all the fish were doubled (or halved)? If we were dealing with ball bearings and rigid round holes, the answer would be obvious; the same fraction of balls would pass through the holes. With fish and fishing nets, the answer is not so clear. Both fish and net are somewhat deformable and fish can force their way through in a tight squeeze. Nevertheless, this only affects fish whose escape is borderline. For those that are substantially too large or too small, the outcome will be the same in the rescaled situation. Thus, on very general grounds, the probability of escape should be similar.This is known as the principle of geometric similarity in the gillnet literature (Baranov, 1914, 1948).

These geometrical arguments suggest that a selectivity model with $I_{50}$ directly proportional to mesh size (or equivalently, constant SF) should be a good first approximation. This gives

$$
\mathrm{I}_{50}=\mathrm{f}(\mathbf{x}) \mathrm{m},
$$

where $f(\mathbf{x})$ is a (dimensionless) function of net variables other than mesh size, such as extension length and codend diameter.

Similar arguments suggest the SR should also be directly proportional to mesh size, or equivalently 
$R F$ be constant. Such arguments are less compelling for $S R$ than for $I_{50}$. The relationship between $I_{50}$ and mesh size is determined mainly by changes in fish girth with fish length; however, the relationship between $S R$ and mesh size is determined mainly by how the deformity of the net changes with mesh size and how the deformity of fish and variation in girth change with fish length. Nevertheless, it is quite reasonable that, as a first approximation,

$$
S R=g(\mathbf{x}) m
$$

where $(\mathbf{x})$ is another function of net variables other than mesh size.

Incorporating these expressions for $I_{50}$ and $S R$ into the logistic selectivity curve:

$$
\log _{e}\left(\frac{p}{1-p}\right)=\frac{2 \log _{e}(3)}{S R}\left(I-I_{50}\right)
$$

gives the 'structural' model:

$$
\log _{e}\left(\frac{p}{1-p}\right)=\frac{2 \log _{e}(3)}{g(x) m}(1-f(\mathbf{x}) m)
$$

This can be simplified by writing $g^{\prime}(\mathbf{x})=2 \log _{e}(3) / g(\mathbf{x})$ and $f^{\prime}(\mathbf{x})=-f^{\prime}(\mathbf{x}) g^{\prime}(\mathbf{x})$ to give:

$$
\log _{e}\left(\frac{p}{1-p}\right)=f^{\prime}(\mathbf{x})+g^{\prime}(\mathbf{x}) \operatorname{lm}{ }^{-1}
$$

This family of structural models, based on geometrical arguments, provides plausible prior models. However, their use must be tempered by several considerations. For example, although mesh size may be doubled, a $50-\mathrm{cm}$ haddock is not exactly double a $25-\mathrm{cm}$ haddock, either in girth, or in wriggling power. Secondly, the geometrical arguments assume that other variables affecting selectivity remain unchanged. However, in practice, changes in mesh size require changes in other net variables, so that the net continues to function properly. For example, a constant codend diameter is maintained by changing the number of meshes round the codend. The latter point is discussed further later on.

A fairly general structural model, incorporating extension length and codend diameters, is:

$$
\begin{gathered}
\text { Model S1 } \log _{e}\left(\frac{p}{1-p}\right)=\alpha_{1}+\alpha_{2} e+\alpha_{3} c+ \\
\left(\alpha_{4}+\alpha_{5} e+\alpha_{6} c\right) \mathrm{lm}^{-1}
\end{gathered}
$$

Similar to models E2, E3 and E0, we also consider:

$$
\text { Model S2 } \log _{e}\left(\frac{p}{1-p}\right)=\alpha_{1}+\alpha_{2} e+\alpha_{3} c+\alpha_{4} \operatorname{Im}^{-1}
$$

$$
\begin{gathered}
\text { Model S3 } \log _{e}\left(\frac{p}{1-p}\right)=\alpha_{1}+\left(\alpha_{4}+\alpha_{5} e+\alpha_{6} c\right) \operatorname{Im}^{-1} \\
\text { Model So } \log _{e}\left(\frac{p}{1-p}\right)=\alpha_{1}+\alpha_{4} \operatorname{Im}^{-1}
\end{gathered}
$$

which are nested within model S1.

These models are interpreted as follows:

S1: Both the selection factor $S F$ and the range factor RF depend on net variables other than mesh size (i.e. codend diameter and extension length), but in different ways.

S2: RF is constant but SF depends on the other net variables.

S3: Both SF and RF depend on the other net variables, but in the same way.

So: Both SF and RF are constant.

Note that a model in which SF is constant and $R F$ depends on the other net variables can be formulated as:

$$
\log _{e}\left(\frac{p}{1-p}\right)=\left(\alpha_{1}+\alpha_{2} e+\alpha_{3} c\right)\left(1+\alpha_{4} I^{-1}\right)
$$

but this model is not considered further here.

\section{Model Fits to Data}

The empirical and structural models were fitted to the selectivity data of Reeves et al. (1992) and Galbraith et al. (1994) using the fixed and random effects model of Fryer (1991). The fitting procedure was in two parts (see Fryer, 1991, for details). A logistic curve was first fitted to the data for each haul. Maximum likelihood was then used to combine these logistic estimates over hauls and hence estimate the parameters $\alpha$. Hauls with very few fish had to be discarded. Generally, plenty of haddock were caught, and few hauls were discarded. However, more whiting hauls and many cod hauls were discarded. (So many cod hauls from the single boat trawl were discarded that no model could be fitted to these data). Thus, the results for haddock should be most 'reliable' and haddock has always been used below for illustrative purposes.

All the models revealed the same qualitative effect of mesh size, extension length and codend diameter on selectivity and gave broadly similar fitted values within the range of experimental data. However, a formal comparison of model fits is not straightforward because the number of parameters varies between models and because the empirical and structural models are not nested. One useful model selection criterion is Akaike's Information Criterion (AIC) (Akaike, 1973, 1974), defined to be: 
$A / C=-2($ maximized log-likelihood $)+2$ (number of estimated parameters).

AlC favours models with good predictive performance, by penalizing models with many parameters that overfit the data. In linear regression models, $A I C$ is an approximately unbiased estimator of the prediction error. For more general models, as here, $A / C$ is asymptotically equivalent to crossvalidation (Stone, 1977), another method of model selection, and considerably easier to compute. As a rule of thumb, models within two units of the lowest $A / C$ are considered plausible 'best' models; of these, the model with the fewest parameters is usually selected (Jones, 1993). Table 1 give the $A / C$ for each model/species/net, scaled so that the minimum $A / C$ is zero.

No model is consistently the best. However, one of the empirical models usually has the lowest $A / C$.
Of these, model E3 appears a good general choice, since it has few parameters and performs reasonably for most data sets. In most cases, one of the structural models also performs reasonably, except for the seine 1 data.

Other model selection criteria, based on crossvalidation statistics, were also considered. These generally supported the conclusions above. Interestingly, a cross-validation statistic based on leaving out each experimental combination (of $m$, $e, c)$ one at a time and predicting the corresponding value of $I_{50}$ virtually mirrored $A / C$ in Table 1. However, the comparable statistic based on predicting $S R$ suggested there was little to choose between any of the models. This suggests that changes in the net variables have a much greater effect of $I_{50}$ than on $S R$ (as noted by Reeves et al., 1992) and that model choice is predominantly determined by the ability to predict $I_{50}$.

TABLE 1. Akaike's information criterion for each model/species/net scaled so that the minimum $A / C$ is zero.

\begin{tabular}{|c|c|c|c|c|c|}
\hline Species & Model & Seine 1 & Seine 2 & Single trawl & Pair trawl \\
\hline \multirow[t]{8}{*}{ Haddock } & EO & 275.1 & 97.4 & 129.6 & 48.2 \\
\hline & SO & 202.6 & 61.6 & 93.6 & 21.3 \\
\hline & E1 & 2.3 & 0.0 & 0.0 & 1.8 \\
\hline & E2 & 11.2 & 20.4 & 17.9 & 1.7 \\
\hline & E3 & 0.0 & 1.1 & 10.2 & 0.0 \\
\hline & S1 & 69.0 & 5.1 & 11.6 & 5.3 \\
\hline & S2 & 65.8 & 6.7 & 22.2 & 3.5 \\
\hline & S3 & 90.0 & 6.9 & 54.9 & 5.7 \\
\hline \multirow[t]{8}{*}{ Whiting } & EO & 138.2 & 91.7 & 96.7 & 31.9 \\
\hline & So & 102.8 & 53.7 & 73.2 & 18.6 \\
\hline & E1 & 0.0 & 0.0 & 0.1 & 2.3 \\
\hline & E2 & 24.0 & 38.4 & 0.0 & 0.0 \\
\hline & E3 & 14.1 & 19.2 & 9.8 & 2.9 \\
\hline & S1 & 15.5 & 3.3 & 17.9 & 3.3 \\
\hline & S2 & 32.2 & 23.5 & 17.0 & 3.1 \\
\hline & S3 & 18.0 & 13.3 & 33.4 & 1.3 \\
\hline \multirow[t]{8}{*}{ Cod } & EO & 218.2 & 109.1 & & 23.5 \\
\hline & So & 154.9 & 58.6 & & 13.6 \\
\hline & E1 & 0.0 & 4.2 & & 1.9 \\
\hline & E2 & 10.1 & 21.0 & & 6.1 \\
\hline & E3 & 2.3 & 0.0 & & 3.8 \\
\hline & S1 & 66.9 & 11.2 & & 0.0 \\
\hline & S2 & 66.7 & 9.0 & & 2.6 \\
\hline & S3 & 85.6 & 28.8 & & 7.7 \\
\hline
\end{tabular}




\section{Interpretation and Application of Model Fits}

Expressions for $I_{50}$ and $S R$ for each model are given in Table 2. By construction, $I_{50}$ and $S R$ are directly proportional to mesh size for the structural models. Various behaviours are possible with model $\mathrm{E} 1$, depending on the values of the parameters $\alpha$. However, for E2, I 50 varies linearly with (but is not generally directly proportional to) mesh size and $S R$ is independent of mesh size. With E3, both $I_{50}$ and $S R$ vary as the inverse of a linear function of mesh size.

Figure 1 shows how $I_{50}$ predictions for haddock depend on the choice of selectivity model as mesh size increases from $80-150 \mathrm{~mm}$. Throughout, an extension length of $9 \mathrm{~m}$ ( $11 \mathrm{~m}$ for the pair trawl) and a codend diameter of $3 \mathrm{~m}$ have been assumed. These are roughly the average values used in the selectivity trials and explain why the $I_{50}$ predictions from the structural models are so similar; real differences between these models only appear at different values of extension length and codend diameter. There is reasonable agreement between the two families of models within the range of experimental mesh sizes $(80-100 \mathrm{~mm}$ for seine 1 , seine 2 and the single trawl and $80-110 \mathrm{~mm}$ for the pair trawl). However, extrapolating outside these mesh sizes causes the $I_{50}$ predictions to diverge. In particular, the $I_{50}$ predictions from models E1 and E3 rapidly increase due to the inverse linear relationship between $I_{50}$ and mesh size. For the data sets considered here, the $I_{50}$ predictions from model E2 increase faster with mesh size than those from the structural models; however, this need not always be the case.

Figure 2 shows how SR predictions for haddock depend on the choice of selectivity model as mesh size increases from $80-150 \mathrm{~mm}$. Again, there is reasonable agreement within the range of experimental mesh sizes, but the $S R$ predictions diverge outside this range. Again, $S R$ predictions from models $E 1$ and $E 3$ increase rapidly with mesh size.

\section{Management Implications}

The choice of an appropriate selectivity model depends on the purpose for which it is to be used. For example, all the models considered here show the effect of mesh size, extension length and codend diameter on the selectivity of the four

\begin{tabular}{c|c|c} 
Model & $I_{50}$ & $S R$ \\
\hline E0 & $-\frac{\alpha_{1}}{\alpha_{5}}$ & $\frac{2 \log _{e}(3)}{\alpha_{5}}$ \\
E1 & $-\frac{\alpha_{1}+\alpha_{2} m+\alpha_{3} e+\alpha_{4} c}{\alpha_{5}+\alpha_{6} m+\alpha_{7} e+\alpha_{8} c}$ & $\frac{2 \log _{e}(3)}{\alpha_{5}+\alpha_{6} m+\alpha_{7} e+\alpha_{8} c}$ \\
E2 & $-\frac{\alpha_{1}+\alpha_{2} m+\alpha_{3} e+\alpha_{4} c}{\alpha_{5}}$ & $\frac{2 \log _{e}(3)}{\alpha_{5}}$ \\
E3 & $-\frac{\alpha_{1}+\alpha_{3} e+\alpha_{4} c}{\alpha_{5}+\alpha_{6} m}$ & $\frac{2 \log _{e}(3)}{\alpha_{5}+\alpha_{6} m}$ \\
\hline S0 & $-\frac{\alpha_{1} m}{\alpha_{4}}$ & $\frac{2 \log _{e}(3) m}{\alpha_{4}}$ \\
S1 & $-\frac{\left(\alpha_{1}+\alpha_{2} e+\alpha_{3} c\right) m}{\alpha_{4}+\alpha_{5} e+\alpha_{6} c}$ & $2 \log _{\ominus}(3) m$ \\
\hline S2 & $-\frac{\left(\alpha_{1}+\alpha_{2} e+\alpha_{3} c\right) m}{\alpha_{4}}$ & $\frac{2 \log _{e}(3) m}{\alpha_{4}+\alpha_{5} \theta+\alpha_{6} c}$ \\
S3 & $-\frac{\alpha_{1} m}{\alpha_{4}+\alpha_{5} e+\alpha_{6} c}$ & $\frac{2 \log _{e}(3) m}{\alpha_{4}+\alpha_{5} e+\alpha_{6} c}$ \\
\end{tabular}



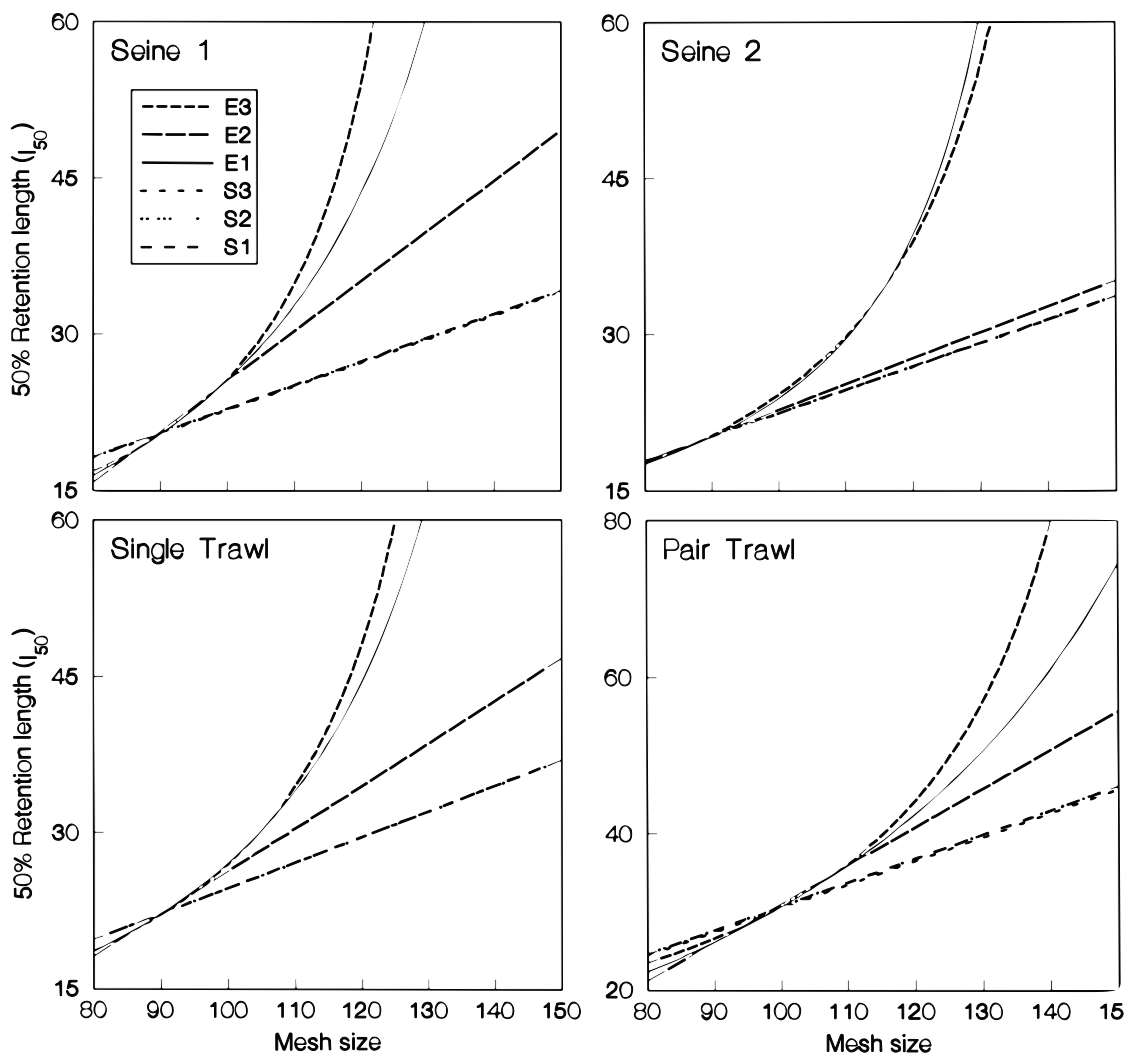

Fig. 1. Haddock $I_{50}$ for mesh sizes between 80 and $150 \mathrm{~mm}$ predicted from models E1-3 and S1-3. Note that the predicted $I_{50}$ for models $S 1-3$ are almost coincident.

experimental nets. Further, to predict selectivity within the range of experimental mesh sizes, extension lengths and codend diameters, a sensible strategy would be to use the 'best' model as determined, for example, by $A / C$. Indeed, for the selectivity trials considered here, all the models would give 'similar' predictions.

Other considerations become important when extrapolating outside the range of experimental data. For example, model E3 often appears a reasonable choice, based on its fit to experimental data. However, extrapolations using this model are highly inconsistent with our intuitive ideas on how a net should behave (see Fig. 1 and 2), so model E3 should not be used for extrapolation. The same applies to the more general model E1 unless the value of the parameter $\alpha_{6}$ is negligible.

As a further example, consider the case of haddock/seine 1. Here, the empirical models fit much better than the structural models. However, some judicious model construction reveals a structural model with a comparable fit; namely:

$$
\log _{e}\left(\frac{p}{1-p}\right)=\alpha_{1}+\alpha_{2} e+\alpha_{3} n+\alpha_{4} \operatorname{lm}^{-1}
$$

when $n$ is the number of meshes round the codend, (highly correlated with the codend diameter). This model suggests that doubling the mesh size, while keeping the extension length and the number of meshes round the codend fixed, would double $I_{50}$. However, suppose that $n=120$ and that mesh size is doubled from 80 to $160 \mathrm{~mm}$. Although $n=120$ and $m=80$ represents a practical net configuration, $n=120$ and $m=160$ would create considerable 'ballooning' of the codend, leading to quite unpredictable selection behaviour. Extrapolations from this model should be viewed with caution.

Despite such cautionary tales, the need to extrapolate remains. In general, the use of a structural model would be recommended when it is the 'best' model according to some empirical model selection criterion. However, an appropriate choice is less clear when, for example, model E2 fits selectivity data better than any of the structural models. It is then necessary to balance the advantages of a geometrically consistent prior 

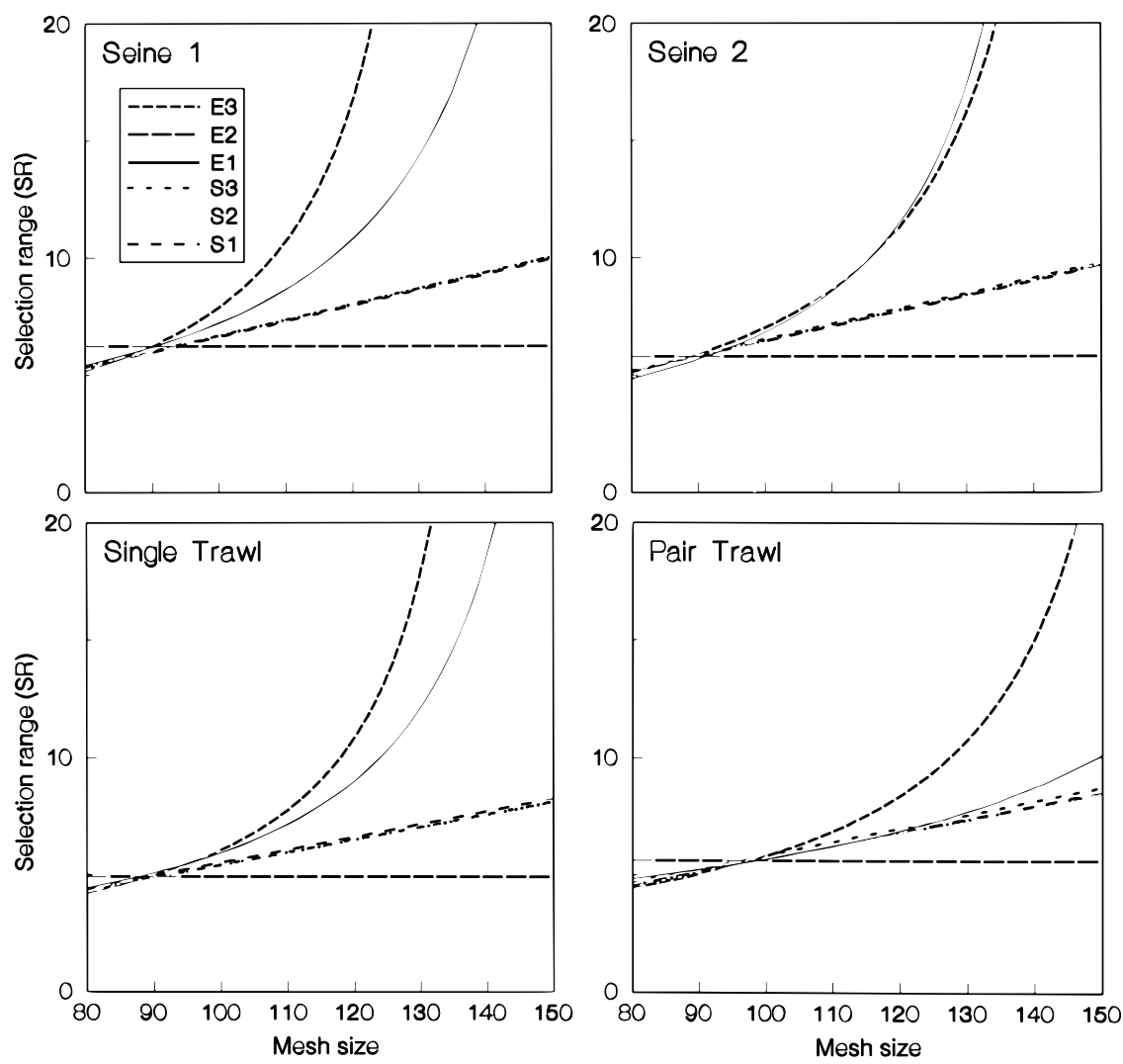

Fig. 2. Haddock $S R$ s for mesh sizes between 80 and $150 \mathrm{~mm}$ predicted from models E1-3 and S1-3. Note that the predicted SRs for models S1-3 are almost coincident.

model with the empirical evidence. One solution would be to present results from both sets of models. If these are close, the extrapolations are insensitive to the choice of model and one can proceed with confidence using either prediction. If the extrapolations differ greatly, the potential dangers arising from uncertainty over the choice of model are made clear. An informed decision can then be made, based on whether one is an empiricist or a structuralist and on any other relevant considerations.

For example, suppose we wish to reduce haddock discards from pair trawls by increasing $I_{50}$ to $40 \mathrm{~cm}$, say, by an appropriate increase in mesh size. From Table 1, models E2 and E3 are competing 'best' models, being within two units of the minimum AlC. Model E2 is preferred to model E3 for extrapolation, and suggests a target mesh size of $118 \mathrm{~mm}$, with an approximate $95 \%$ confidence interval (conditional on model E2 being correct) of 114-126 mm. Model S2 also has a pretty good fit and gives a target mesh size of $130 \mathrm{~mm}$ with a $95 \%$ confidence interval of 125-136 $\mathrm{mm}$. The two predictions do not completely conflict, given that the confidence intervals overlap. A general estimation method would be to take a weighted average of the two predictions, with weights based on a) the strength of our prior preference for a structural model and $b$ ) the difference between the two empirical fits. However, the question of an appropriate weighting function immediately arises. A more satisfactory solution would be to look at the short- and long-term implications of each model choice, assuming each is the correct model, and under mutual model mis-specification. In the example above, what would be the biological, social and economic consequences of an increase in mesh size to $130 \mathrm{~mm}$ if $I_{50}$ increases to $40 \mathrm{~cm}$ as predicted by model $\mathrm{S} 2$ or to $46 \mathrm{~cm}$ as predicted by model E2? Similarly, what would be the effect of an increase in mesh size to $118 \mathrm{~mm}$ ? Such an approach falls naturally into the philosophy of risk analysis, increasingly being used in fisheries management.

Ideally, of course, extrapolation should be used to design cost-effective selectivity trials to 
investigate proposed changes in net variables. Uncertainties over model choice can be accommodated in the design of the trial. For example, a trial could investigate the range of mesh sizes that models E2 and S2 suggest might give the target $I_{50}$. The only remaining uncertainty is then the familiar one of relating experimental studies to commercial operations.

When extrapolation is necessary, a good principle is to choose models that give sensible predictions outside the range of experimental variables. On this principle, structural models will often be preferred, since extrapolation using empirical models can, except in special cases, lead to unrealistic predictions of $I_{50}$ and $S R$ for reasonable values of mesh size.

\section{Acknowledgements}

We thank Claire Ross for helping compute various cross-validation statistics and two anonymous referees for their helpful comments and suggestions.

\section{References}

AKAIKE, H. 1973. Information theory and an extension of the maximum likelihood principle. Second International Symposium on Information Theory, (B.N. Petrov and F. Csaki, Ed.), Budapest. Akademia Kaido, 267-281.

1974. A new look at statistical model identification. IEEE Trans. on Automatic Control, AC-19, 716-723.

BARANOV, F. I. 1914. The capture of fish by gillnets. Poznsniyu. Russ. Rybolovsta. 3: 56-99. (Translation from Russian in 1976 Selected works on fishing gear. Vol. 1. Commercial fishing techniques. Israel programme for scientific translations, Jerusalem.)

BARANOV, F. I. 1948. Theory and assessment of fishing gear. Ch. 7, Theory of fishing with gillnets. Pishchepromizdat, Moscow. (Translation from Russian by Ontario Dept. of Lands For., Maple, Ont., 45 p.)

FENDER, D. H. 1957. General physics and sound to advanced and scholarship level. English Universities Press, London, $415 \mathrm{p}$.

FRYER, R. J. 1991. A model of between-haul variation in selectivity. ICES J. Mar. Sci., 48: 281-290.

GALBRAITH, D. K., R. J. FRYER, and K. M. S. MAITLAND. 1994. Demersal pair trawl cod-end selectivity models. Fish. Res., 20: 13-27.

GILCHRIST, W. 1984. Statistical modelling. John Wiley and Sons, Chichester, $339 \mathrm{p}$.

JONES, R. H. 1993. Longitudinal data with serial correlation: a state-space approach. Chapman and Hall, London. 225 p.

MILLAR, R. B. MS 1991. Estimation of asymmetric selection curves for trawls. ICES C. M. Doc., No. B: $56,21 \mathrm{p}$.

1992. Estimating the size-selectivity of fishing gear by conditioning on the total catch. J. Amer. Stat. Assoc., 87: 962-968.

1993. Incorporation of between-haul variation using bootstrapping and nonparametric estimation of selection curves. Fish. Bull. U.S., 91: 564-572.

1994. Sampling from trawl gears used in size selectivity experiments. ICES J. Mar. Sci., 51: 293298.

MILLAR, R. B., and S. J. WALSH. 1992. Analysis of trawl selectivity studies with an application to trouser trawls. Fish. Res., 13: 205-220.

POPE, J. A., A. R. MARGETTS, J. M. HAMLEY, and E. F. AKYUZ. 1975. Manual of methods for fish stock assessment. Part 3 - selectivity of fishing gears. FAO Fish. Tech. Pap., No. 41(Rev. 1), 65 p.

REEVES, S. A., D. W. ARMSTRONG, R. J. FRYER, and K. A. COULL. 1992. The effects of mesh size, cod-end extension length and cod-end diameter on the selectivity of Scottish trawls and seines. ICES J. Mar. Sci., 49: 279-288.

STONE, M. 1977. An asymptotic equivalence of choice of model by cross-validation and Akaike's criterion. J. Royal. Statist. Soc., B39: 44-47. 